\title{
The Design of a Computer Language for Linguistic Information
}

\author{
Stuart M. Shieber \\ Artificial Intelligence Center \\ SRI International \\ and \\ Center for the Study of Language and Information \\ Stanford University
}

\begin{abstract}
A considerable body of accumulated knowledge about the design of languages for communicating information to computers has been derived from the subfields of programming language design and semantics. It has been the goal of the PATR group at SRI to utilize a relevant portion of this knowledge in implementing tools to facilitate communication of linguistic information to computers. The PATR-II formalism is our current computer language for encoding linguistic information. This paper, a brief overview of that formalism, attempts to explicate our design decisions in terms of a set of properties that effective computer languages should incorporate.
\end{abstract}

\section{Introduction ${ }^{1}$}

The goal of natural-language processing research can be stated quite simply: to endow computers with human language capability. The pursuit of this objective, however, has been a difficult task for at least two reasons: first, this capability is far from being a well-understood phenomenon; second, the tools for teaching computers what we do know about human language are still very primitive. The solution of these problems lies within the respective domains of linguistics and computer science.

Similar problems have arisen previously in computer science. Whenever a new computer application area emerges, there follow new modes of communication with computers that are geared towards such areas. Computer languages are a direct result of this need for effective communication with computers. A considerable body of accumulated knowledge about the design of languages for communicating information to computers has been derived from the subfields of programming language design and seman-

'This research has been made possible in part by a gift trom the Systems Development Foundation, and was also supported by the Defense Advanced Research Projects Agency under Contract N00039-80-C0575 with the Naval Electrodic Systems Command. The views and conclusions contained in this document are those of the author and stould not be interpreted as representative of the official policies, ejther expressed or implied, of the Defense Advanced Research Projects Agency, or the United States government.

The author is indebted to Fernando Pereira, Barbara Grosz, and Ray Perrault for their comments on earlier drafts. tics. It has been the goal of the PATR group at SRI' to utilize a relevant portion of this knowledge in implementing tools to facilitate communication of linguistic information to computers.

The PATR-I formalism is our current computer language for encoding linguistic information. This paper, a brief overview of that formalism, attempts to explicate our design decisions in terms of a set of properties that effective computer languages should incorporate, namely: simplicity, power, mathematical well-foundedness, flexibility, implementability, modularity, and declarativeness. More extensive discussions of various aspects of the PATR-II formalism and systems can be found in papers by Shieber et al., [83], Pereira and Shieber [84] and Karttunen [84].

The notion of designing specialized computer languages and systems to encode linguistic information is not new; PROGRAMMAR [Winograd, 72], ATNs [Woods, 70], and DIALOGIC [Grosz, et al., 82] are but a few of the better-known examples. Furthermore, a trend has arisen recently in linguistics towards declarativeness in grammar formalisms - for instance, lexical-functional grammar (LFG) [Bresnan, 83], generalized phrase-structure grammar (GPSG) [Gazdar and Pullum, 82] and functional unification grammar (UG) [Kay, 83]. Finally, in computer science there has been a great deal of interest in declarative languages (e.g., logic programming and specification languages), and their supporting denotational semantics. But to our knowledge, no attempt has yet been made to combine the three approaches so as to yield a declarative computer language with clear semantics designed specifically for encoding linguistic information. Such a language, of which PATR-II is an example, would reflect a felicitous convergence of ideas from linguistics, artificial intelligence, and computer science.

\section{The Critical Properties of the Language}

It is not the purpose of this paper to provide a comprehensive description of the PATR-II project, or even of the formalism itself. Rather, we will discuss briefly the critical ${ }^{2}$ This rather liquid group has included at various times: John Bear, Lauri Karttunen, Fernando Pereira, Jane Robinson, Stan Rosenschein, Susan Stucky, Mabry Tyson, Hans Uszkoreit, and the author. 
properties of PATR-II to give a flavor for our approach to the design of the language. References to papers with more complete descriptions of particular aspects of the project are provided when appropriate.

\subsection{Simplicity: An Introduction to the PATR-II Formalism}

Building on a convergence of ideas from the linguistics and AI communities, PATR-II takes as its primitive operation an extended pattern-matching technique, unification, first used in logic and theorem-proving research and lately finding its way into research in linguistics (Kay, 79; Gazdar and Pullum, 82] and knowledge representation [Reynolds, 70; Ait-Kaci, 83]. Instead of unifying logic terms, however, PATR unification operates on directed acyclic graphs (DAG). ${ }^{3}$

DAGs can be atomic symbols or sets of label/value pairs, where the values are themselves DAGs (either atomic or complex). Two labels can have the same value-thus the use of the term graph rather than tree. DAGs are notated either by drawing the graph structure itself, with the labels marking the arcs, or, as in this paper, by notating the sets of label/value pairs in square brackets, with the labels separated from their values by a colon; e.g., a DAG associated with the verb "knight" (as in "Uther wants to knight Arthur") would appear (in at least one of our grammars) as

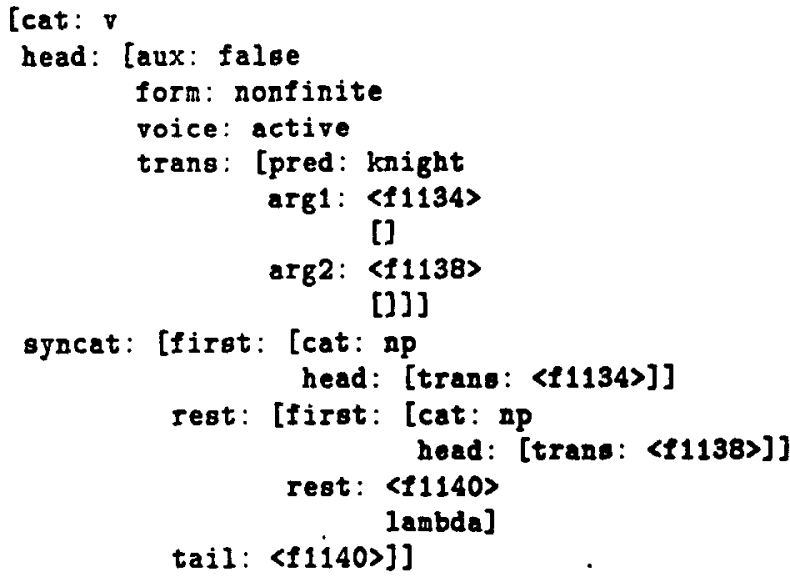

Reentrant structure is notated by labeling the DAG with an arbitrary label (in angle brackets), then using that label for future references to the DAG.

Associated with each entry in the lexicon is a set of DAGs. The root of each DAG will have an arc labeled cat ${ }^{3}$ Technically, these are rooted, directed, acyclic graphs with labeled arcs. Formal definition of these and otber technical notions can be found in Appendix $A$ ol Shieber et al. [83]. Note that some implementations have been extended to handle cyclic graph structures as well as graph structures with disjunction and negation |Karttunen, 84].

'In our implementation, this association is not directly encoded-since this would yield a grossly inefficient characterization of the lexiconbut is mediated by a morphological analyzer. See Section 2.6 for further details. whose value will be the category of the associated lexical entry. Other arcs may encode information alout the syntactic features, translation, or syntactic subcategorization of the entry. But only the label cal has any special significance; it provides the link between context-free phrase structure rules and the DAGs, as explicated below.

PATR-II grammars consist of rules with a context-free phrase structure portion and a sct of unifications on the DAGs associated with the constituents that participate in the application of the rule. The grammar rules describe how constituents can be built up to form new constituents with associated DAGs. The right side of the rule lists the cat values of the DAGs associated with the filial constituents; the left side, the cat of the parent. The associated unifications specify equivalences that must exist among the various DAGs and sub-DAGs of the parent and children. Thus, the formalism uses only one representation - DAGsfor lexical, syntactic, and semantic information, and one operation--unification-on this representation.

By way of example, we present a trivial grammar for a fragment of English with a lexicon associating words with DAGs.

$$
\begin{aligned}
S \rightarrow N P V P & \\
<V P \text { agr }> & =
\end{aligned}
$$

This grammar (plus lexicon) adnits the two sentences "Uther knights Arthur" and "Arthur knights Uther." The phrase structure associated with the first of these is:

[s [NP Uther] [vP [v knights] [NP Arthur]]]

The VP rule requires that the agr feature of the DAG associated with the VP be the same as (unified with) the agr of the V. Thus, the VP's agr feature will have as its value the same node as the V's agr, and hence the same values for the person and number features. Similarly, by virtue of the unification associated with the S rule, the NP will have the same agr value as the VP and, consequently, the V. We have thus encoded a form of subject-verb agreement.

Note that the process of unification is order-independent. For instance, we would get the same effect regardless of whether the unifications at the top of the parse tree were effected before or after those at the bottom. In either case, the DAG associated with, e.g., the VP node would be 


$$
\begin{aligned}
& \text { [cat: vp } \\
& \text { agr: [person: third } \\
& \quad \text { number: singular]] }
\end{aligned}
$$

These trivial examples of grammars and lexicons offer but a glimpse of the techniques used in writing PATR-II grammars, and do not begin to employ the power of unification as a general information-passing mechanism. Examples of the use of PATR.II for encoding much more complex linguistic phenomena can be found in Shieber et al. [83].

\subsection{Power: Two Variants}

Augmented phrase-structure grammars such as PATRII can in lart be quite powerful. The ability to encode unbuunderl amounts of information in the augmentations (which PIIT-II obviously allows) gives this formalism the power of a Turing machine. As a linguistic theory, this much power might be considered disadvantageous; as a romputer language, however, such power is clearly desiralile. since the intent of the language is to enable the modcling of many kinds of linguistic analyses from a range of theorics. Is surh, PATR-II is a tool, not a result.

Nevertheless, a good case could be made for maintaining at least the decidability of determining whether a string is admitted by a PATR-II grammar. This property can be ensured by requiring the context-free skeleton to have the property of off-line parsability [Pereira, 83], which was used originally in the definition of LFG to maintain the decidability of that formalism [Kaplan and Bresnan, 83]. Off-line parsability requires that the context-free "skeleton" of the grammar allows no trivial cyclic derivations of the form $A \dot{\doteq} A$.

\subsection{Mathematical Well-Foundedness: Denotational Semantics}

One reason for maintaining the simplicity of the bare PATR-II formalism is to permit a clean semantics for the language. We have provided a denotational semantics for PATR-II [Percira and Shieber, 84] based on the information systems domain theory of Dana Scott [Scott, 82]. Insofar as more complex formalisms, such as GPSG and LFG, can be modelerl as appropriate notations for PATR-II grammars, PATR-Il's denotational semantics constitutes a framework in which the semantics of these formalisms can also be defined, discussed, and compared. As it appears that not all the power of domain theory is needed for the semantics of PATR-II, we are currently pursuing the possibility of building a semantics based on a less powerful model. ${ }^{5}$

\subsection{Flexibility: Modeling Linguistic Con- structs}

Clearly, the bare PATR-II formalism, as it was presented in Section 2.1, is sorely inadequate for any major attempt at building natural-language grammars because of its verbosity and redundancy. Efficiency of encoding was ${ }^{3}$ But sce Pereira and Shieber [84| for arguments in favor of using domain theory even if all the available power is not utilized. temporarily sacrificed in an attempt to keep the underlying formalism simple, general, and somantically well-founded. However, given a simple underlying formalism, we can build more efficient, specialized languages on top of it, much as MACLISP might be built on top of pure LISP. And just as MACLISP need not be implemented (and is not implemented) directly in pure LISP, specialized formalisms built conceptually on top of pure PATR-II need not be so implemented (although currently we do implement them directly through pure PATR-II). The effectiveness of this approach can be seen in the fact that at least a sizable portion of English syntax has been encoded in various experimental PATR-II grammars constructed to date. The syntactic constructs encoded include subcategorization of various complement types ( $N P, \bar{S}$ s, etc.), active, passive, "there" insertion, extraposition, raising, and equi-NP constructions, and unbounded dependencies (such as Wh-movencent and relative clauses). Other theory-dependent devices that have been modeled with PATR-II include head-feature percolation [Gazdar and Pullum, 82], and LFG-like semantic forms [Kaplan and Bresnan, 83]. Note that none of these constructs and techniques required expansion of the underlying formalism; indeed, the constructions all make use of the techniques described in this section. See Shicber et al. [83] for a detailed discussion of the modeling of some of these phenomena.

The devices now available for molding PATR-II to conform to a particular intended usage or linguistic theory are in their nascent stage. However, because of their great importance in making the PATR-II system a usalile one, we will discuss them briefly. It is important to keep in mind that these methods should not be considered a part of the underlying formalism, but merely "syntactic sugar" to increase the system's utility and allow it to conform to a user's intentions.

\subsubsection{Templates}

Because so much of the information in the PATR-II grammars under actual development tends to be encoded in the lexicon, most of our research has been devoted to methods for removing redundancy in the lexicon by alluw. ing the users themselves to define primitive constructs and operations on lexical items. Primitive constructs, such as the transitive, dyadic, or equi-NP properties of a verb, can be defined by means of templates, that is, DAG that encode some linguistically isolable portion of the DAG of a lexical item. These template DAGs can then be conbined to build the lexical item out of the user-defined prinitives.

As a simple example, we could define (with the following syntax) the template Verb as

Let Verb be

$$
<c a t\rangle=V
$$

and the template ThirdSing as

$$
\begin{aligned}
& \text { Let ThirdSing be } \\
& \text { <agr number> = singular } \\
& \text { <agr person }>=\text { third }
\end{aligned}
$$

The lexical entry for "knights" would then be 
knights:

\section{Verb ThirdSing}

Templates can themselves refer to other templates, enabling definition of abstract linguistic concepts hierarchically. For instance, a modal verb template may use an auxiliary verb template, which in term may be defined using the verb template above. In fact, templates are currently employed for abstracting notions of subcategorization, verb form, semantic type, and a host of other concepts.

\subsubsection{Lexical Rules}

More complex relationships among lexical items can be encoded by means of lexical rules. These rules, such as passive and "there" insertion, are user-definable operations on the lexical itcms, enabling one variant of a word to be built from the specification of another variant. A lexical rule is specified as a set of selective unifications relating an input DAG and an output DAG. Thus, unification is the primitive used in this device as well.

Lexical rules are used to encode the relationships among various lexical entries that would typically be thought of as transformations or relation-changing rules (depending on one's ideological outlook). Because lexical rules perform these operations, the lexicon need include only a prototype entry for each verb. The variant forms can be derived through lexical rules applied in accordance with the morphology actually found on the verb. (The morphological analysis in the implementations of PATR-II is performed by a program based on the system of Koskenniemi [83] and was written by Lauri Karttunen [83].)

For instance, given a PATR-II grammar in which the DAGs are used to emulate the f-structures of LFG, we might write a passive lexical rule as follows (following Bresnan $(83]):$

$$
\begin{aligned}
\text { Define Passive as } & \\
<\text { out cat }> & =\text { <in cat> } \\
<\text { out form }> & =\text { passprt } \\
<\text { out subj }> & =\text { <in obj> } \\
<\text { out obj }> & =<\text { in subj> }
\end{aligned}
$$

The rule states in effect that the output DAG (the one associated with the passive verb form) marks the lexical item as being a passive verb whose object is the input DAG's subject and whose subject is the input's object. Such lexical rules have been used for encoding the active/passive dichotomy, "there" insertion, extraposition, and other socalled relation-changing rules.

\subsection{Modularity and Declarativeness}

The PATR-II formalism is a completely declarative formalism, as evidenced by its denotational semantics and the order-independence of its definition. Modularity is achieved through the ability to define primitive templates and lexical rules that are shared among lexical items, as well as by the declarative nature of the grammar formalism itself,

${ }^{6}$ The example is merely meant to be indicative of the syntax for and operation of lexical rules. We do not present this as a valid definition of Passive for any grammar we have written in PATR-Il. removing problems of interaction of rules. Rules are guaranteed to always mean the same thing, regardless of the environment of other rules in which they are placed.

\subsection{Implementability}

Implementability is an empirical matter, given credence by the fact that we now have three implementations of the formalism. One desirable aspect of the simplicity and declarative nature of the formalism is that even though the three implementations differ substantially from one another, using different parsing algorithrns (with both top down and bottom up properties), different implementations of unification, different methods of compiling the rules, all are able to run on exactly the same grammars yielding the identical results.

The three implementations of the PATR-II system currently in operation at SRI are as follows:

- An INTERLISP version for the DEC-2060 using a variant of the Cocke-Kasami-Younger parsing algorithm and the KIMMO morphological analyzer [Karttunen, 83], and a limited programming environment.

- A ZETALISP version for the Symbolics 3600 using a left-corner parsing algorithm and the KIMMO morphological analyzer, with an extensive programming environment (due primarily to Mabry Tyson) that includes incremental compilation, multiple window debugging facilities, tracing, and an integrated editor.

- A Prolog version (DEC-10 Prolog) running on the DEC-2060 by Fernando Pereira, designed primarily as a testbed for experimentation with eflicient structuresharing DAG unification algorithms, and incorporating an Earley-style parsing algorithm.

In addition, Lauri Karttunen and his students at the University of Texas have implemented a system based on PATR-II but with several interesting extensions, including disjunction and negation in the graph structures /liarttunen, 84]. These extensions will undoubtedly be integrated into the SRI systems and formal semantics for them are being pursued.

\section{Conclusion}

The PATR-II formalism was designed as a computer language for encoding linguistic information. The design was influenced by current theory and practice in computer science, and especially in the areas of programming language design and semantics. The formalism is simple (consisting of just one primitive operation, unification), powerful (although it can be constrained to be decidable), mathematically well-founded (with a complete denotational semantics), flexible (as demonstrated by its ability to model analyses in GPSG, LFG, DCG and other formalisms), modular (because of its higher-level notational devices such as templates and lexical rules), declarative (yielding orderindependence of operations), and implementable (as demonstrated by three quite dissimilar implemented systems and one highly developed programming environment). 
As we have omphasized herein, PATR-II seems to represent a connromre of techniques from several domainscomputer scicnce, programming language design, natural language processing and linguistics. Its positioning at the center of these tronds arises, however, not from the admixture of many discrote techniques, but rather from the application of a single simple yet powerful concept to the encoding of linguistic information.

\section{References}

Ait-Kari, 11., 198:: "A new Model of Computation Based on a Calculus of 'Type Subsumption," Doctoral Dissertation Proposal, Dept. of Computer and Information Science, University of Pennsylvania (Novenber).

Bresnan, Joan, 109\%: The montal representation of grammatical rclations (cd.), Cambrirlor: MIT Press.

Gazdar, Ci and CiK. Pullum, 1982: "GPSG: A Theoretical Synopsis," Indiana University Linguistics Club, Bloomington, lndiana.

Grosz, B., N. Haan, C: llemlrix, J. Hobbs, P. Martin, R. Moore,

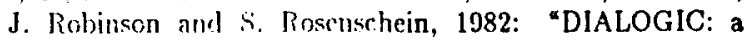
core natural-langunise processing system," Proceedings of the Ninth Intcrnational Conference on Computational Linguis. lics, Prague, Czechoslavakia (July), pp. 95-100.

Kaplan, R. and J. Bressian, 1983: "Lexical-Functional Grammar: A Formal System for Grammatical Representation," in J. Bresnan (ect.). The mental representation of grammatical relutions (rd.), (ambridge: MIT Press.

Karttunen, L., 198.1: "Fentures and Values," Proceedings of the Tenth International Conference on Computational Linguistics, Stanford Iniversity, Stanford California (4.7 July, 1984).

Karttunen, L.., 1983: "KIMMO: a general morphological processor," Texus Linguistic Forum, Volume 22 (December), pp. 161-185.

Kay, M., 1979: "Funclinnal Cirammar," in Proceedings of the Fiflh Annual Mceting of the Berkeley Linguistics Society, Berkeley, California (17-19 February).

hay, M., 1083: "Unification Grammar," unpublished memo, Xerox Palo Alto Research Center.

Koskenniemi, K., 1083: “A Two level Model for Morphological Analysis and Synthesis," fortheoming Ph.D. dissertation, University of Iledsinki, Ilelsinki, Finland.

Percira, F. and D.Il.D. Warren, 1983: “Parsing as Deduction," in Procerdings of the 1st Anmual Meeting of the Association for Computationul lingnistics (15-17 June), pp. 137-144.

Percira, F. and S. Shicher, 1984: "The Semantics of Grammar Formalisms Scen as Computer Languages," Proceedings of the Tenth Intcrnational Conference on Computational Lin. guistics, Stanford University, Stanford California (4-7 July, 1984).

Reynolds, J., 1070: "Transformational Systems and the Algebraic Structure of Atomic Formulas," in D. Michie (ed.), Machine Intellijence, Vol. 5, Chapter 7, Edinburgh, Scotland: Edinburgh University Press, pp. 135-151.

Scott, D., 1982: "Domains for Denotational Semantics," ICALP '82, Aarhus, Denmark (July).
Shieber, S., H. Uszkoreit, F. Percira, J. Robinson, and M. Tyson, 1983: "The Formalism and Implementation of PATR-II," in B. Grosz and M. Stickel, Research on Interactive Acquisition and Use of Knowledge, SRI Final Report 1894, SRI International, Menlo Park, California (November).

Winograd, T., 1972: Understanding Natural Lanyuage, New York, New York: Academic Press.

Woods, W., 1970: “Transition Network Grammars for Natural Language Analysis," Communications of the ACM, Vol. 13, No. 10 (October). 